\title{
Ionic liquid-mediated three-component synthesis of fluorinated spiro-thiazine derivatives and their antimycobacterial and DNA cleavage activities
}

\author{
ANSHU DANDIA*, RUBY SINGH and DEEPTI SAINI \\ Department of Chemistry, University of Rajasthan, Jaipur 302 004, India \\ e-mail: dranshudandia@yahoo.co.in
}

MS received 19 December 2012; revised 14 May 2013; accepted 18 June 2013

\begin{abstract}
A simple, green and catalyst-free novel protocol is developed for the synthesis of medicinally important spiro[indole-3,2' [1,3]-thiazine]-2,4'-dione and spiro[acenaphthylene-1,2' -[1,3] thiazine]dione libraries by the tandem reaction of readily available reagents in 1-butyl-3-methylimidazolium hexafluorophosphate $[\mathrm{bmim}]\left[\mathrm{PF}_{6}\right]$. The ionic liquid has been used as a solvent as well as catalyst for this reaction. This reaction proceeded smoothly in good to excellent yields and offered several other advantages including short reaction time, simple experimental workup procedure and no by-products. The synthesized compounds were subjected to antimycobacterial efficacy against Mycobacterium tuberculosis H37Rv strain and DNA cleavage activity.
\end{abstract}

Keywords. Environmentally benign; spiro-thiazine derivative; tandem reaction; antimycobacterial activity; DNA scavenging activity.

\section{Introduction}

Deoxyribonucleic acid (DNA) is the intracellular target for wide range of anticancer and antibiotic drugs. ${ }^{1}$ Studies on interaction of drug molecules with DNA have become an active research in recent years as they facilitate molecular interaction studies which may result in devising new drugs with different mechanisms and models of action. Despite recent progress in cancer chemotherapy, high toxicity and low specificity of current medications are motivating scientists to search for safer and more effective anticancer drugs. ${ }^{2}$ Metal-based molecules are the foremost and widely used anticancer drugs in cancer therapy, ${ }^{3}$ but these possess inherent side effects, solubility and acquired drug resistance. Therefore, considerable attempts are being made to replace these drugs with suitable alternatives.

Thia-azaheterocycles have attracted considerable attention because of their wide biological and pharmacological activities. ${ }^{4}$ Moreover, thia-azaheterocycles exhibited potent antitumour activities against non-small cell lung cancer cell line H460, paclitaxel-resistant H460 taxR, human colon cancer cell line HT-29 and human breast cancer cell line MDA-MB-231. ${ }^{5}$ Thiazine and its derivatives are an important class of hete-

*For correspondence rocyclic compounds possessing broad biological activities, such as COX-1 inhibition, ${ }^{6}$ antiinflammatory, ${ }^{7}$ antiproliferative, ${ }^{8}$ antihistaminic, ${ }^{9}$ and anti-HIV activities. ${ }^{10}$ These are also known as anti-radiation agents and used as radiation-sickness drugs. ${ }^{11}$ Furthermore, antibiotic activity of cephalosporins is due to the presence of 1,3-thiazine nucleus. ${ }^{12}$ As regards chemical viewpoint, 1,3-thiazines are important synthetic intermediates in organic syntheses. ${ }^{13}$

Organo fluorine compounds have been receiving significant attention in materials and pharmaceutical sciences due to their unique physical and biological properties such as the increased membrane permeability, enhanced hydrophobic binding and stability against metabolic oxidation. ${ }^{14}$ Among these compounds, trifluoromethyl group-containing molecules are especially important, and continue to attract increasing attention from various fields. ${ }^{15}$ Since fluorine is virtually absent in the living tissue, ${ }^{16}$ fluorinated pharmaceuticals might possess comparatively less environmental and mammalian toxicity.

Indole derivatives constitute an important class of therapeutic agents in medicinal chemistry including anticancer, ${ }^{17}$ antioxidant, ${ }^{18}$ antirheumatoidal and anti$\mathrm{HIV}^{19}$ properties and also play a vital role in the immune system. ${ }^{20}$ Spirooxindole possesses a myriad of biological activities such as inhibition of the mammalian cell cycle at $\mathrm{G} 2 / \mathrm{M}$ phase, ${ }^{21}$ inhibition of 
microtubule assembly, ${ }^{22}$ modulation of the function of muscarinic serotonin receptors, ${ }^{23}$ antitumour activity against human brain cancer cell lines, neuroplastoma SKN-BE, and malignant glioma GAMG. ${ }^{24}$ In addition, spiroindoles containing thia-azaheterocyclic ring system as a structural motif are present in many pharmacologically important synthetic and naturally occurring compounds (as typified by spirobrassinin). ${ }^{25}$

The above-mentioned biological importance inspired us to attach spirooxindoles to the thiazine scaffold, and the combination of two privileged structures in one molecule leads to drug-like molecules.

A number of methods have been reported for the preparation of spirooxindole derivatives involving the synthon thioacids. ${ }^{26}$ All these processes use plenty of organic solvents, reactions have suffered from long reaction time and a narrow scope of substrates. The spiro[indole-3,2' $[1,3]$-thiazine]-2,4'-diones incorporating two biodynamic heterocyclic thiazine and indole moieties ${ }^{27}$ through a spiro carbon atom appear to be of great interest and were earlier synthesized by reacting 3-indolylimine (intermediate such as Shiff-base formed by condensation of isatin and aniline) and 3mercaptopropionic acid using high boiling carcinogenic hydrocarbons with continuous azeotropic removal of water. ${ }^{28}$ Therefore, it is necessary to develop an efficient and new versatile method for the synthesis of these compounds.

Multi-component reactions (MCRs) are of increasing importance in organic and medicinal chemistry, because the strategies of MCR offer significant advantages over conventional linear-type syntheses. ${ }^{29}$ MCRs leading to interesting heterocyclic scaffolds are particularly useful for the creation of diverse chemical libraries of 'drug-like' molecules for biological screening, since the combination of three or more small molecular weight building blocks in a single operation leads to a high combinatorial efficacy.
In recent years, ionic liquids (ILs) have received recognition as green media in organic synthesis due to the ease of tuning their physical properties, such as good solvating capability, wide liquid range, negligible vapour pressure, tunable polarity, high thermal stability and ease of recyclability. ${ }^{30}$ The rising number of publications is indicative of the potential of ILs as 'designer solvents' for various chemical reactions.

Considering the fact that ILs have been used as useful and efficient media for esterification reactions in spite of the 'in situ' formation of water ${ }^{31}$ because they can create an opportunity to drive the equilibrium and in line with our interest on spiro indoles, ${ }^{32}$ we studied the possibility of employing the easily made 1,3-dialkylimidazolium cation-based IL as green and efficient solvent for the preparation of spiro[indole3,2' [1,3]-thiazine]-2,4'-dione by three-component reaction of isatin 1, aniline $\mathbf{2}$ and mercaptopropionic acid 3 in IL without any catalyst (scheme 1). We were also interested in knowing the probable impact of assembling of 1,3-thiazine with oxindoles moiety in search of novel anticancer agents through DNA cleavage procedure.

\section{Experimental}

Melting points of all compounds were determined on a Toshniwal apparatus. Purity of compounds was checked on thin layers of silica Gel-G coated glass plates and n-hexane: ethyl acetate (8:2) as eluent. IR spectra were recorded on a Shimadzu FT IR-8400S spectrophotometer using $\mathrm{KBr}$ pellets. ${ }^{1} \mathrm{H}$ and ${ }^{13} \mathrm{C}$ NMR spectra were recorded in DMSO- $d_{6}$ and $\mathrm{CDCl}_{3}$ using tetramethylsilane (TMS) as an internal standard on a Bruker Avance spectrophotometer at 300 and $75 \mathrm{MHz}$, respectively. Mass (ESI) spectra of compounds were recorded on JEOL SX-102 spectrometer at $70 \mathrm{eV}$. Elemental analyses were carried out on a Carlo-Erba $1108 \mathrm{CHN}$ analyzer.

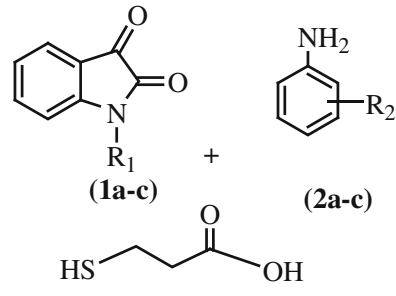

(3)

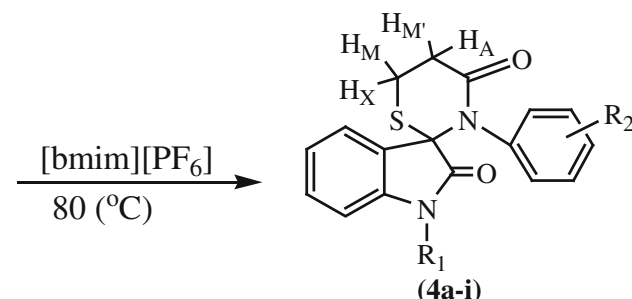

(4a-i)

\begin{tabular}{c|ccc}
$\mathrm{R}_{1}$ & $\mathrm{CH}_{2}-\mathrm{C}_{6} \mathrm{H}_{5}(\mathbf{1 a})$ & $\mathrm{CH}_{2}-\mathrm{CH}=\mathrm{CH}_{2}(\mathbf{1 b})$ & $-\mathrm{C}_{2} \mathrm{H}_{5}(\mathbf{1 c})$ \\
\hline $\mathrm{R}_{2}$ & $4-\mathrm{Cl} \mathbf{( 2 a )}$ & $3-\mathrm{F}(\mathbf{2 b})$ & $3-\mathrm{CF}_{3}(\mathbf{2} \mathbf{c})$
\end{tabular}

Scheme 1. Synthesis of spiro[indole-3,2' $[1,3]$-thiazine $]-2,4^{\prime}$-diones $(\mathbf{4 a - i})$. 
2.1 Syntheses of spiro[indole-3,2' [1,3]-thiazine]-2, $4^{\prime}$-diones (4a-i) and spiro[acenaphthylene-1,2' [1,3]thiazine]diones (6a-f)

A mixture of appropriate indole-2,3-dione (1) / acenaphthalene-1,2-dione (5) $(1 \mathrm{mmol})$, aniline $(2)(1 \mathrm{mmol})$ and 3-mercaptopropionic acid (3) $(1.5 \mathrm{mmol})$ and ionic liquid, [bmim] $\mathrm{PF}_{6}(2 \mathrm{~mL})$ were taken in a conical flask. Contents of the flask were stirred magnetically at $80^{\circ} \mathrm{C}$. After completion of the reaction (as monitored by thin layer chromatography (TLC)), the product was extracted with diethyl ether and ethereal extracts were evaporated to give a crystalline material. The pure products were characterized by spectral data $\left({ }^{1} \mathrm{H}\right.$ nuclear magnetic resonance (NMR), ${ }^{13} \mathrm{C}$ NMR and Mass). ${ }^{1} \mathrm{H}$ NMR spectra of $\mathbf{4 a}-\mathbf{i}$ and $\mathbf{6 a}-\mathbf{f}$ showed three sets of multiplets due to thiazine ring protons which can be explained according to an first order spectra $\left(\mathrm{AMM}^{\prime} \mathrm{X}\right)$ splitting pattern (schemes 1 and 2), indicating different environment for $\mathrm{H}_{\mathrm{A}}$ and $\mathrm{H}_{\mathrm{X}}$ protons. Similar environment for $\mathrm{H}_{\mathrm{M}}$ and $\mathrm{H}_{\mathrm{M}^{\prime}}$ protons, with little difference in chemical shift, meant that their signals could not be separated and a complex multiplet integrating for two protons appeared. Spectral data for some products are given below.

\subsection{Characterization of compounds $(\mathbf{4} \mathbf{a}-\mathbf{i})$}

2.2a 1-Benzyl-3'(4-chlorophenyl)-spiro[indole-3,2' [1,3]thiazine $]-2,4^{\prime}(1 H)$-dione $(\mathbf{4 a})$ : $\mathrm{IR}\left(\mathrm{KBr}, v, \mathrm{~cm}^{-1}\right)$ 1695, $1710(\mathrm{C}=\mathrm{O}) ;{ }^{1} \mathrm{H}$ NMR $(300 \mathrm{MHz}, \mathrm{DMSO}-$ $\left.d_{6}\right) \delta(\mathrm{ppm}): 2.64-2.76\left(\mathrm{~m}, 1 \mathrm{H}, \mathrm{H}_{\mathrm{A}}\right), 3.18-3.31(\mathrm{~m}, 2 \mathrm{H}$, $\left.\mathrm{H}_{\mathrm{MM}^{\prime}}\right), 4.16-4.26\left(\mathrm{~m}, 1 \mathrm{H}, \mathrm{H}_{\mathrm{X}}\right), 4.89(\mathrm{~d}, 1 \mathrm{H}, \mathrm{CH}, J=$ $15.6 \mathrm{~Hz})$ and $5.10(\mathrm{~d}, 1 \mathrm{H}, \mathrm{CH}, J=15.6 \mathrm{~Hz}), 6.74$ $7.32(\mathrm{~m}, 13 \mathrm{H}, \mathrm{Ar}-\mathrm{H}) ;{ }^{13} \mathrm{C}$ NMR $\left(75 \mathrm{MHz}, \mathrm{DMSO}-d_{6}\right)$ $\delta(\mathrm{ppm}): 29.4,32.3,54.90,78.58,119.01,121.95$, $123.57,124.56,125.05,125.78,127.68,128.58$, $129.09,129.99,131.58,134.01,138.23,142.68$, 168.16, 176.09; MS (ESI, $\mathrm{m} / z, \mathrm{M}^{+}$): 434.0; Anal. calcd. for $\mathrm{C}_{24} \mathrm{H}_{19} \mathrm{ClN}_{2} \mathrm{O}_{2} \mathrm{~S}$ : C, 66.28; $\mathrm{H}, 4.40 ; \mathrm{N}, 6.44 \%$. Found; C, 66.12; H, 4.35; N, 6.39\%.

2.2b 1-Benzyl-3'(3-fluorophenyl)-spiro[indole-3,2' [1,3]thiazine $]-2,4^{\prime}(1 \mathrm{H})$-dione $(\mathbf{4 b})$ : $\mathrm{Mp} \quad 274-276^{\circ} \mathrm{C}$; IR $\left(\mathrm{KBr}, \quad v, \quad \mathrm{~cm}^{-1}\right) \quad 1690,1715 \quad(\mathrm{C}=\mathrm{O}) ;{ }^{1} \mathrm{H} \quad \mathrm{NMR}$ $\left(300 \mathrm{MHz}, \mathrm{DMSO}-d_{6}\right) \delta(\mathrm{ppm}): 2.63-2.77\left(\mathrm{~m}, 1 \mathrm{H}, \mathrm{H}_{\mathrm{A}}\right)$,
3.17-3.33 (m, 2H, $\left.\mathrm{H}_{\mathrm{MM}^{\prime}}\right), 4.19-4.28\left(\mathrm{~m}, 1 \mathrm{H}, \mathrm{H}_{\mathrm{X}}\right), 4.92$ $(\mathrm{d}, 1 \mathrm{H}, \mathrm{CH}, J=15.6 \mathrm{~Hz})$ and $5.14(\mathrm{~d}, 1 \mathrm{H}, \mathrm{CH}, J=$ $15.6 \mathrm{~Hz}), \quad 6.72-7.35$ (m, $13 \mathrm{H}, \quad$ Ar- $\mathrm{H}) ;{ }^{13} \mathrm{C}$ NMR $\left(75 \mathrm{MHz}\right.$, DMSO- $d_{6}$ ) $\delta(\mathrm{ppm}): 28.48,32.30,56.99$, 78.58, 117.24, 119.34, 120.91, 122.09, 124.01, 125.98, $127.58,128.73,129.09,130.89,131.59,132.19,133.08$, 141.68, 142.32, 154.69, 168.56, 176.68; MS (ESI, $\mathrm{m} / \mathrm{z}$, $\mathrm{M}^{+}$): 418.1; Anal. calcd. for $\mathrm{C}_{24} \mathrm{H}_{19} \mathrm{FN}_{2} \mathrm{O}_{2} \mathrm{~S}$ : C, 68.88; $\mathrm{H}, 4.58 ; \mathrm{N}, 6.69 \%$. Found: C, 68.73; H, 4.51; N, 6.62\%.

2.2c 1-Benzyl-3'(3-trifluoromethylphenyl)-spiro[indole3,2'[1,3]-thiazine]-2,4'(1H)-dione (4c): Mp 250$252^{\circ} \mathrm{C}$; IR $\left(\mathrm{KBr}, v, \mathrm{~cm}^{-1}\right)$ 1690, $1713(\mathrm{C}=\mathrm{O}) ;{ }^{1} \mathrm{H}$ NMR $\left(300 \mathrm{MHz}, \mathrm{DMSO}-d_{6}\right) \delta(\mathrm{ppm}): 2.68-2.77(\mathrm{~m}$, $\left.1 \mathrm{H}, \mathrm{H}_{\mathrm{A}}\right), 3.19-3.35\left(\mathrm{~m}, 2 \mathrm{H}, \mathrm{H}_{\mathrm{MM}^{\prime}}\right), 4.18-4.30(\mathrm{~m}$, $\left.1 \mathrm{H}, \mathrm{H}_{\mathrm{X}}\right), 4.91(\mathrm{~d}, 1 \mathrm{H}, \mathrm{CH}, J=15.5 \mathrm{~Hz}), 5.13(\mathrm{~d}$, $1 \mathrm{H}, \mathrm{CH}, J=15.5 \mathrm{~Hz}), 6.76-7.38(\mathrm{~m}, 13 \mathrm{H}, \mathrm{Ar}-\mathrm{H})$; ${ }^{13} \mathrm{C}$ NMR (75 MHz, DMSO- $d_{6}$ ) $\delta(\mathrm{ppm}): 29.67,32.60$, $58.65,81.58,117.01,119.37,120.90,123.70,124.67$, $126.70,127.60,127.80,128.30,129.0,130.0,131.20$, $133.58,138.59,141.89,142.83,142.40,169.02$, 177.69; MS (ESI, $m / z, \mathrm{M}^{+}$): 468.1; Anal. calcd. for $\mathrm{C}_{25} \mathrm{H}_{19} \mathrm{~F}_{3} \mathrm{~N}_{2} \mathrm{O}_{2} \mathrm{~S}$ : C, 64.09; H, 4.09; N, 5.98\% Found: $\mathrm{C}, 64.22 ; \mathrm{H}, 4.02 ; \mathrm{N}, 5.92 \%$.

2.2d 1-Allyl-3'(4-chlorophenyl)-spiro[indole-3,2'tetrahydro-1,3-thiazine $]-2,4^{\prime}(1 \mathrm{H})$-dione $\quad(\mathbf{4 d})$ : $\mathrm{Mp}$ 254-256 ${ }^{\circ}$; IR $\left(\mathrm{KBr}, v, \mathrm{~cm}^{-1}\right)$ 1690, $1712(\mathrm{C}=\mathrm{O}) ;{ }^{1} \mathrm{H}$ NMR $\left(300 \mathrm{MHz}\right.$, DMSO- $\left.d_{6}\right) \delta(\mathrm{ppm}): 2.62-2.75(\mathrm{~m}$, $\left.1 \mathrm{H}, \mathrm{H}_{\mathrm{A}}\right), 3.19-3.28\left(\mathrm{~m}, 2 \mathrm{H}, \mathrm{H}_{\mathrm{MM}^{\prime}}\right), 4.10-4.22(\mathrm{~m}, 1 \mathrm{H}$, $\left.\mathrm{H}_{\mathrm{X}}\right), 4.29\left(\mathrm{dd}, 1 \mathrm{H}, \mathrm{CH}_{2}, J_{1}=16.1 \mathrm{~Hz}, J_{2}=7.9 \mathrm{~Hz}\right)$, $4.58\left(\mathrm{dd}, 1 \mathrm{H}, \mathrm{CH}_{2}, J_{1}=16.1 \mathrm{~Hz}, J_{2}=7.9 \mathrm{~Hz}\right), 5.25$ $\left(\mathrm{d}, 1 \mathrm{H}, \mathrm{CH}_{2}, J=4.4 \mathrm{~Hz}\right), 5.43\left(\mathrm{~d}, 1 \mathrm{H}, \mathrm{CH}_{2}, J=\right.$ $4.4 \mathrm{~Hz}), 5.84$ (m, 1H, CH), 6.72-7.23 (m, 8H, Ar-H); ${ }^{13} \mathrm{C}$ NMR $\left(75 \mathrm{MHz}, \mathrm{DMSO}-d_{6}\right) \delta(\mathrm{ppm}): 29.32,32.15$, 56.97, 77.80, 118.01, 120.90, 121.80, 124.67, 127.60, $128.09,129.99,130.09,131.58,134.58,138.90$, 140.68, 167.16, 178.09; MS (ESI, $m / z, \mathrm{M}^{+}$): 384.1; Anal. calcd. for $\mathrm{C}_{20} \mathrm{H}_{17} \mathrm{ClN}_{2} \mathrm{O}_{2} \mathrm{~S}$ : C, 62.41; H, 4.45; N, 7.28\% Found: C, 62.23; H, 4.39; N, 7.22\%.

2.2e 1 -Allyl-3'(3-fluorophenyl)-spiro[indole-3,2'tetrahydro-1,3-thiazine ]-2,4'(1H)-dione $\quad(\mathbf{4 e})$ : $\mathrm{Mp}$ 255-257 ${ }^{\circ} \mathrm{C}$; IR $\left(\mathrm{KBr}, v, \mathrm{~cm}^{-1}\right)$ 1695, $1715(\mathrm{C}=\mathrm{O}) ;{ }^{1} \mathrm{H}$

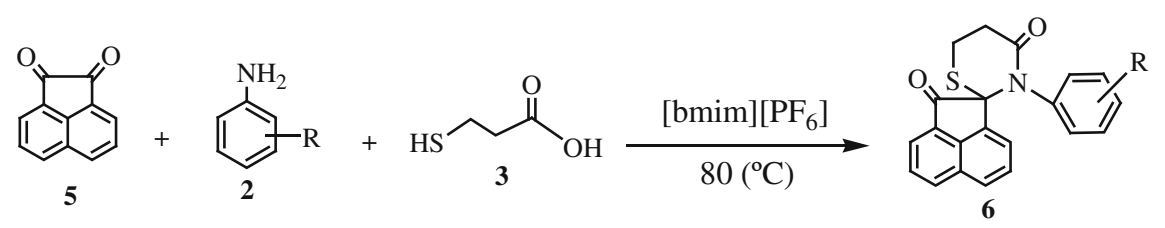

Scheme 2. Synthesis of spiro[acenaphthylene-1,2'-[1,3]thiazine]-2,4'-diones. 
NMR $\left(300 \mathrm{MHz}, \mathrm{DMSO}-d_{6}\right) \delta(\mathrm{ppm}): 2.64-2.76(\mathrm{~m}$, $\left.1 \mathrm{H}, \mathrm{H}_{\mathrm{A}}\right), 3.18-3.31\left(\mathrm{~m}, 2 \mathrm{H}, \mathrm{H}_{\mathrm{MM}^{\prime}}\right), 4.16-4.26(\mathrm{~m}, 1 \mathrm{H}$, $\left.\mathrm{H}_{\mathrm{X}}\right), 4.35\left(\mathrm{dd}, 1 \mathrm{H}, \mathrm{CH}_{2}, J_{1}=14.4 \mathrm{~Hz}, J_{2}=8.1 \mathrm{~Hz}\right)$, $4.54\left(\mathrm{dd}, 1 \mathrm{H}, \mathrm{CH}_{2}, J_{1}=14.4 \mathrm{~Hz}, J_{2}=8.1 \mathrm{~Hz}\right), 5.35$ $\left(\mathrm{d}, 1 \mathrm{H}, \mathrm{CH}_{2}, J=4.3 \mathrm{~Hz}\right), 5.48\left(\mathrm{~d}, 1 \mathrm{H}, \mathrm{CH}_{2}, J=\right.$ $4.3 \mathrm{~Hz}), 5.99(\mathrm{~m}, 1 \mathrm{H}, \mathrm{CH}), 6.74-7.32(\mathrm{~m}, 8 \mathrm{H}, \mathrm{Ar}-\mathrm{H})$; ${ }^{13} \mathrm{C}$ NMR (75 MHz, DMSO- $\left.d_{6}\right) \delta(\mathrm{ppm}): 29.33,32.17$, 58.91, 78.80, 111.10, 116.01, 120.90, 122.36, 124.67, $126.58,127.60,128.09,129.39,130.09,131.54$, 138.90, 140.68, 142.41, 166.16, 175.09; MS (ESI, $\mathrm{m} / \mathrm{z}$, $\mathrm{M}^{+}$): 368.1; Anal. calcd. for $\mathrm{C}_{20} \mathrm{H}_{17} \mathrm{FN}_{2} \mathrm{O}_{2} \mathrm{~S}$ : C, 65.20; H, 4.65; N, 7.60\% Found: C, 65.1 0; H, 4.59; N, 7.56\%.

$2.2 \mathrm{f} \quad 1$-Allyl-3'(3-trifluoromethylphenyl)-spiro[indole3,2' [1,3]-thiazine $]-2,4^{\prime}(1 H)$-dione $(\mathbf{4 f}): \quad \mathrm{Mp} \mathrm{260-}$ $262^{\circ} \mathrm{C}$; IR $\left(\mathrm{KBr}, v, \mathrm{~cm}^{-1}\right)$ 1695, $1713(\mathrm{C}=\mathrm{O}) ;{ }^{1} \mathrm{H}$ NMR $\left(300 \mathrm{MHz}, \mathrm{DMSO}-d_{6}\right) \delta(\mathrm{ppm}): 2.56-2.70(\mathrm{~m}$, $\left.1 \mathrm{H}, \mathrm{H}_{\mathrm{A}}\right), 3.17-3.22\left(\mathrm{~m}, 2 \mathrm{H}, \mathrm{H}_{\mathrm{MM}^{\prime}}\right), 4.21-4.26(\mathrm{~m}, 1 \mathrm{H}$, $\left.\mathrm{H}_{\mathrm{X}}\right), 4.31\left(\mathrm{dd}, 1 \mathrm{H}, \mathrm{CH}_{2}, J_{1}=14.2 \mathrm{~Hz}, J_{2}=8.0 \mathrm{~Hz}\right)$, $4.52\left(\mathrm{dd}, 1 \mathrm{H}, \mathrm{CH}_{2}, J_{1}=14.2 \mathrm{~Hz}, J_{2}=8.0 \mathrm{~Hz}\right), 5.28$ $\left(\mathrm{d}, 1 \mathrm{H}, \mathrm{CH}_{2}, J_{1}=3.2 \mathrm{~Hz}\right), 5.48\left(\mathrm{~d}, 1 \mathrm{H}, \mathrm{CH}_{2}\right), 5.89$ $(\mathrm{m}, 1 \mathrm{H}, \mathrm{CH}), 6.98-7.43(\mathrm{~m}, 8 \mathrm{H}, \mathrm{Ar}-\mathrm{H}) ;{ }^{13} \mathrm{C}$ NMR $\left(75 \mathrm{MHz}, \mathrm{DMSO}-d_{6}\right) \delta(\mathrm{ppm}): 28.90,31.91,57.58$, $80.58,117.23,119.34,120.90,121.22,122.84,123.36$, $126.58,128.32,129.39,130.09,131.54,134.64$, 139.21, 138.90, 142.41, 166.16, 175.09; MS (ESI, $\mathrm{m} / \mathrm{z}$, $\mathrm{M}^{+}$): 418.1; Anal. calcd. for $\mathrm{C}_{21} \mathrm{H}_{17} \mathrm{~F}_{3} \mathrm{~N}_{2} \mathrm{O}_{2} \mathrm{~S}$ : C, 60.28; H, 4.10; N, 6.69\% Found: C, 60.12; H, 4.06; N, 6.62\%.

$2.2 \mathrm{~g}$ 1-Ethyl-3'(4-Chlorophenyl)-spiro[indole-3,2' [1,3]-thiazine]-2,4' $(1 \mathrm{H})$-dione $(\mathbf{4 g}): \quad \mathrm{Mp} 275-277^{\circ} \mathrm{C}$; IR $\left(\mathrm{KBr}, v, \mathrm{~cm}^{-1}\right) 1690,1715(\mathrm{C}=\mathrm{O}) ;{ }^{1} \mathrm{H}$ NMR $\left(300 \mathrm{MHz}, \mathrm{DMSO}-d_{6}\right) \delta(\mathrm{ppm}): 1.33(\mathrm{t}, 3 \mathrm{H}, J=$ $6.8 \mathrm{~Hz}), \quad 2.62-2.70\left(\mathrm{~m}, 1 \mathrm{H}, \mathrm{H}_{\mathrm{A}}\right), 3.28-3.39(\mathrm{~m}$, $\left.2 \mathrm{H}, \mathrm{H}_{\mathrm{MM}^{\prime}}\right), 3.85(\mathrm{q}, 2 \mathrm{H}, J=6.8 \mathrm{~Hz}), 4.26-4.29$ $\left(\mathrm{m}, 1 \mathrm{H}, \mathrm{H}_{\mathrm{X}}\right), 6.94-7.32(\mathrm{~m}, 8 \mathrm{H}, \mathrm{Ar}-\mathrm{H}) ;{ }^{13} \mathrm{C} \mathrm{NMR}$ $\left(75 \mathrm{MHz}, \mathrm{DMSO}-d_{6}\right) \delta(\mathrm{ppm}): 11.33,28.41,30.32$, $55.73,87.58,119.01,120.50,123.16,124.29,126.98$, 129.99, 130.59, 132.24, 133.89, 138.90, 167.28, 176.09; MS (ESI, $\mathrm{m} / \mathrm{z}, \mathrm{M}^{+}$): 372.0; Anal. calcd. for $\mathrm{C}_{19} \mathrm{H}_{17} \mathrm{ClN}_{2} \mathrm{O}_{2} \mathrm{~S}: \mathrm{C}, 61.28 ; \mathrm{H}, 4.40 ; \mathrm{N}, 6.44 \%$. Found: C, 61.08; H, 4.44; N, 6.41\%.

2.2h 1-Ethyl-3'(3-fluorophenyl)-spiro[indole-3,2' [1,3]thiazine $]-2,4^{\prime}(1 H)$-dione $(\mathbf{4 h}): \quad \mathrm{Mp} \quad 270-272^{\circ} \mathrm{C}$; IR $\left(\mathrm{KBr}, v, \mathrm{~cm}^{-1}\right) 1690,1710(\mathrm{C}=\mathrm{O}) ;{ }^{1} \mathrm{H} \mathrm{NMR}(300 \mathrm{MHz}$, DMSO- $\left.d_{6}\right) \delta(\mathrm{ppm}): 1.34(\mathrm{t}, 3 \mathrm{H}, J=6.8 \mathrm{~Hz}), 2.69-2.73$ $\left(\mathrm{m}, 1 \mathrm{H}, \mathrm{H}_{\mathrm{A}}\right), 3.24-3.42\left(\mathrm{~m}, 2 \mathrm{H}, \mathrm{H}_{\mathrm{MM}^{\prime}}\right), 3.88(\mathrm{q}, 2 \mathrm{H}$, $J=6.8 \mathrm{~Hz}), 4.29-4.32\left(\mathrm{~m}, 1 \mathrm{H}, \mathrm{H}_{\mathrm{X}}\right), 6.84-7.32(\mathrm{~m}, 8 \mathrm{H}$, Ar-H); ${ }^{13} \mathrm{C}$ NMR (75 MHz, DMSO- $\left.d_{6}\right) \delta(\mathrm{ppm}): 11.83$, 29.42, 32.36, 57.73, 89.58, 118.29, 120.22, 122.16,
$123.39,124.58,125.42,126.98,128.99,130.59$, 131.54, 134.67, 139.90, 169.16, 177.09; MS (ESI, $\mathrm{m} / \mathrm{z}$, $\mathrm{M}^{+}$): 356.1; Anal. calcd. for $\mathrm{C}_{19} \mathrm{H}_{17} \mathrm{FN}_{2} \mathrm{O}_{2} \mathrm{~S}$ : C, 60.28; H, 4.41; N, 6.24\%. Found: C, 60.42; H, 4.46; N, 6.19\%.

$2.2 \mathrm{i}$ 1-Ethyl-3'(3-trifluoromethylphenyl)-spiro[indole-3,

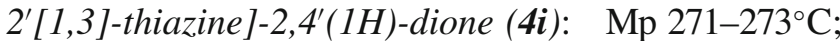
IR $\left(\mathrm{KBr}, v, \mathrm{~cm}^{-1}\right) 1695,1715(\mathrm{C}=\mathrm{O}) ;{ }^{1} \mathrm{H}$ NMR $\left(300 \mathrm{MHz}\right.$, DMSO- $\left.d_{6}\right) \delta(\mathrm{ppm}): 1.23(\mathrm{t}, 3 \mathrm{H}, J=$ $6.5 \mathrm{~Hz}), 2.66-2.72\left(\mathrm{~m}, 1 \mathrm{H}, \mathrm{H}_{\mathrm{A}}\right), 3.26-3.36(\mathrm{~m}, 2 \mathrm{H}$, $\left.\mathrm{H}_{\mathrm{MM}^{\prime}}\right), 3.88(\mathrm{q}, 2 \mathrm{H}, J=6.5 \mathrm{~Hz}), 4.20-4.25(\mathrm{~m}, 1 \mathrm{H}$, $\left.\mathrm{H}_{\mathrm{X}}\right), 6.98-7.32(\mathrm{~m}, 8 \mathrm{H}, \mathrm{Ar}-\mathrm{H}) ;{ }^{13} \mathrm{C}$ NMR $(75 \mathrm{MHz}$, DMSO- $\left.d_{6}\right) \delta(\mathrm{ppm}): 12.33,28.12,31.36,58.73,81.58$, $119.01,121.36,122.50,123.16,124.66,125.98$, $126.39,127.99,130.59,131.54,138.90,142.41$, 144.29, 166.90, 175.09; MS (ESI, $\mathrm{m} / z, \mathrm{M}^{+}$): 406.1. Anal. calcd. for $\mathrm{C}_{20} \mathrm{H}_{17} \mathrm{~F}_{3} \mathrm{~N}_{2} \mathrm{O}_{2} \mathrm{~S}: \mathrm{C}, 61.28 ; \mathrm{H}, 4.40 ; \mathrm{N}$, 6.44\% Found: C, 61.24; H, 4.45; N, 6.40\%.

\subsection{Characterization of compounds $(\mathbf{6 a - f})$}

2.3a 3'(2-Fluorophenyl)-spiro[acenaphthylene-1,2' [1,3]thiazine $]-2,4^{\prime}(1 H)$-dione $(\boldsymbol{6 a})$ : $\mathrm{Mp} \quad 257-259^{\circ} \mathrm{C}$; IR $\left(\mathrm{KBr}, v, \mathrm{~cm}^{-1}\right) 1700,1715(\mathrm{C}=\mathrm{O}) ;{ }^{1} \mathrm{H}$ NMR $\left(300 \mathrm{MHz}, \mathrm{DMSO}-d_{6}\right) \delta(\mathrm{ppm}): 2.59-2.68(\mathrm{~m}, 1 \mathrm{H}$, $\left.\mathrm{H}_{\mathrm{A}}\right), 3.22-3.35\left(\mathrm{~m}, 2 \mathrm{H}, \mathrm{H}_{\mathrm{MM}^{\prime}}\right), 4.25-4.33(\mathrm{~m}, 1 \mathrm{H}$, $\left.\mathrm{H}_{\mathrm{X}}\right), 7.02-7.73(\mathrm{~m}, 10 \mathrm{H}, \mathrm{Ar}-\mathrm{H}) ;{ }^{13} \mathrm{C}$ NMR $(75 \mathrm{MHz}$, DMSO- $\left.d_{6}\right) \delta(\mathrm{ppm}): 25.7,35.9,94.9,115.70,121.50$, $123.16,124.89,126.98,127.99,128.59,129.01$, $129.89,130.59,131.54,132.79,133.08,133.68$, 138.90, 154.41, 175.09, 192.89; MS (ESI, $\mathrm{m} / z, \mathrm{M}^{+}$): 363.0; Anal. calcd. for $\mathrm{C}_{21} \mathrm{H}_{14} \mathrm{FNO}_{2} \mathrm{~S}$ : C, 69.41; H, 3.88 ; N, 3.85\%. Found: C, 69.30; H, 3.82; N, 3.79\%.

$2.3 \mathrm{~b} \quad 3$ '(3-Trifluoromethylphenyl)-spiro[acenaphthylene1,2'[1,3]-thiazine]-2,4'(1H)-dione $(\boldsymbol{6 b})$ : Mp 227$229^{\circ} \mathrm{C}$; IR $\left(\mathrm{KBr}, v, \mathrm{~cm}^{-1}\right) 1700,1720(\mathrm{C}=\mathrm{O}) ;{ }^{1} \mathrm{H}$ NMR (300 MHz, DMSO- $\left.d_{6}\right) \delta(\mathrm{ppm}): 2.60-2.70\left(\mathrm{~m}, 1 \mathrm{H}, \mathrm{H}_{\mathrm{A}}\right)$, 3.24-3.34(m, 2H, $\left.\mathrm{H}_{\mathrm{MM}^{\prime}}\right), 4.34-4.39\left(\mathrm{~m}, 1 \mathrm{H}, \mathrm{H}_{\mathrm{X}}\right), 7.10$ $7.74\left(\mathrm{~m}, 10 \mathrm{H}\right.$, Ar-H); ${ }^{13} \mathrm{C}$ NMR $\left(75 \mathrm{MHz}, \mathrm{DMSO}-d_{6}\right)$ $\delta(\mathrm{ppm}): 24.12,34.33,95.90,117.24,119.35,120.90$, $121.50,123.16,124.32,126.98,127.99,129.01$, $129.81,130.52,131.50,132.23,133.02,133.56$, 138.88, 141.68, 174.09, 192.65; MS (ESI, $\mathrm{m} / \mathrm{z}, \mathrm{M}^{+}$): 413.0; Anal. calcd. for $\mathrm{C}_{22} \mathrm{H}_{14} \mathrm{~F}_{3} \mathrm{NO}_{2} \mathrm{~S}$ : C, 63.92; $\mathrm{H}$, 3.41 ; N, 3.39\%. Found: C, 63.85; H, 3.32; N, 3.34\%.

2.3c 3'(4-Chloro,3-trifluoromethylphenyl)-spiro [acenaphthylene-1,2' [1,3]-thiazine]-2,4'(1H)-dione $(\mathbf{6 c})$ : Mp 145-147 ${ }^{\circ} \mathrm{C}$; IR (KBr, $\left.v, \mathrm{~cm}^{-1}\right)$ 1705, $1685(\mathrm{C}=\mathrm{O})$; ${ }^{1} \mathrm{H}$ NMR (300 MHz, DMSO- $\left.d_{6}\right) \quad \delta(\mathrm{ppm}): 2.50-2.60$ 
$\left(\mathrm{m}, 1 \mathrm{H}, \mathrm{H}_{\mathrm{A}}\right), 3.04-3.14\left(\mathrm{~m}, 2 \mathrm{H}, \mathrm{H}_{\mathrm{MM}^{\prime}}\right), 4.14-4.22$ $\left(\mathrm{m}, 1 \mathrm{H}, \mathrm{H}_{\mathrm{X}}\right), 7.02-7.64(\mathrm{~m}, 9 \mathrm{H}, \mathrm{Ar}-\mathrm{H}) ;{ }^{13} \mathrm{C}$ NMR $\left(75 \mathrm{MHz}, \mathrm{DMSO}-d_{6}\right) \delta(\mathrm{ppm}): 24.02,34.03,94.20$, $110.23,117.24,121.50,123.16,125.13,126.25$, $127.99,128.01,129.89,130.59,131.54,132.79$, 133.08, 133.68, 139.68, 142.39, 150.21, 175.09, 192.65; MS (ESI, $\mathrm{m} / \mathrm{z}, \mathrm{M}^{+}$): 447.0; Anal. calcd. for $\mathrm{C}_{22} \mathrm{H}_{13} \mathrm{ClF}_{3} \mathrm{NO}_{2} \mathrm{~S}: \mathrm{C}, 59.00 ; \mathrm{H}, 2.93 ; \mathrm{N}, 3.13 \%$. Found: C, 58.84; H, 2.84; N, 3.08\%.

$2.3 \mathrm{~d} \quad 3^{\prime}$ (4-Fluorophenyl)-spiro[acenaphthylene-1,2' [1,3]-thiazine]-2,4'(1H)-dione (6d): $\mathrm{Mp} 193-195^{\circ} \mathrm{C}$; IR $\left(\mathrm{KBr}, v, \mathrm{~cm}^{-1}\right) 1705,1715(\mathrm{C}=\mathrm{O}) ;{ }^{1} \mathrm{H}$ NMR $\left(300 \mathrm{MHz}, \mathrm{DMSO}-d_{6}\right) \delta(\mathrm{ppm}): 2.60-2.65(\mathrm{~m}, 1 \mathrm{H}$, $\left.\mathrm{H}_{\mathrm{A}}\right), 3.40-3.44\left(\mathrm{~m}, 2 \mathrm{H}, \mathrm{H}_{\mathrm{MM}^{\prime}}\right), 4.24-4.32(\mathrm{~m}, 1 \mathrm{H}$, $\left.\mathrm{H}_{\mathrm{X}}\right), 7.02-7.68\left(\mathrm{~m}, 10 \mathrm{H}\right.$, Ar-H) ${ }^{13} \mathrm{C}$ NMR $(75 \mathrm{MHz}$, DMSO- $\left.d_{6}\right) \quad \delta(\mathrm{ppm}): 25.01, \quad 34.03$, 94.90, 115.23, $123.10,125.13,126.25,126.98,127.99,128.01$, $128.98,129.89,130.59,131.54,132.79,133.08$, 140.28, 172.09, 190.65; MS $(\mathrm{m} / \mathrm{z})$ : 363.0; Anal. calcd. for $\mathrm{C}_{21} \mathrm{H}_{14} \mathrm{FNO}_{2} \mathrm{~S}$ : C, 69.25; H, 3.88; N, 3.85\%. Found: C, 69.31; H, 3.82; N, 3.74\%.

2.3e 3'(4-Methoxyphenyl)-spiro[acenaphthylene-1,2' [1,3]-thiazine]-2,4' $(1 \mathrm{H})$-dione (6e): $\mathrm{Mp} 131-133^{\circ} \mathrm{C}$; IR $\left(\mathrm{KBr}, v, \mathrm{~cm}^{-1}\right) 1695,1705(\mathrm{C}=\mathrm{O}) ;{ }^{1} \mathrm{H}$ NMR (300 MHz, DMSO- $\left.d_{6}\right) \delta(\mathrm{ppm}): 2.66-2.70\left(\mathrm{~m}, 1 \mathrm{H}, \mathrm{H}_{\mathrm{A}}\right)$, 3.29-3.38 (m, 2H, $\left.\mathrm{H}_{\mathrm{MM}^{\prime}}\right), 4.20-4.26\left(\mathrm{~m}, 1 \mathrm{H}, \mathrm{H}_{\mathrm{X}}\right), 3.65$ $\left(\mathrm{s}, 3 \mathrm{H}, \mathrm{OCH}_{3}\right), 6.82-7.78(\mathrm{~m}, 10 \mathrm{H}, \mathrm{Ar}-\mathrm{H}) ;{ }^{13} \mathrm{C} \mathrm{NMR}$ $\left(75 \mathrm{MHz}, \mathrm{DMSO}-d_{6}\right) \delta(\mathrm{ppm}): 25.70,34.89,56.09$, 93.90, 114.23, 121.24, 123.10, 125.03, 126.50, 127.99, $128.01,128.98,129.89,130.59,131.54,132.79$, 134.08, 142.08, 173.09, 190.65; MS (ESI, $\mathrm{m} / z, \mathrm{M}^{+}$): 375.0; Anal. calcd. for $\mathrm{C}_{22} \mathrm{H}_{17} \mathrm{NO}_{3} \mathrm{~S}$ : C, 70.38; $\mathrm{H}, 4.56$; N, 3.73\% Found: C, 70.22; H, 4.50; N, 3.68\%.

$2.3 \mathrm{f} \quad 3$ (4-Chlorophenyl)-spiro[acenaphthylene-1,2' [1,3]-thiazine]-2, $4^{\prime}(1 \mathrm{H})$-dione $(\boldsymbol{6 f}): \quad \mathrm{Mp} 205-207^{\circ} \mathrm{C}$; IR $\left(\mathrm{KBr}, v, \mathrm{~cm}^{-1}\right) 1700,1715(\mathrm{C}=\mathrm{O}) ;{ }^{1} \mathrm{H}$ NMR (300 MHz, DMSO- $\left.d_{6}\right) \delta(\mathrm{ppm}): 2.63-2.70\left(\mathrm{~m}, 1 \mathrm{H}, \mathrm{H}_{\mathrm{A}}\right)$, 3.34-3.40 (m, 2H, $\left.\mathrm{H}_{\mathrm{MM}^{\prime}}\right)$, 4.35-4.43 (m, $\left.1 \mathrm{H}, \mathrm{H}_{\mathrm{X}}\right), 7.02-$ 7.68 (m, 10H, Ar-H); ${ }^{13} \mathrm{C}$ NMR (75 MHz, DMSO- $\left.d_{6}\right)$ $\delta(\mathrm{ppm}): 25.79,35.96,92.92,118.19,121.50,123.16$, $124.89,126.98,127.99,128.59,129.01,129.49$, $130.89,131.54,133.68,138.90,141.42,171.09$, 190.22; MS (ESI, $\mathrm{m} / \mathrm{z}, \mathrm{M}^{+}$): 379.0; Anal. calcd. for $\mathrm{C}_{21} \mathrm{H}_{14} \mathrm{ClNO}_{2} \mathrm{~S}: \mathrm{C}, 66.40 ; \mathrm{H}, 3.71 ; \mathrm{N}, 3.69 \%$. Found: C, 66.28; H, 3.42; N, 3.62\%.

\section{Results and discussion}

\subsection{Chemistry}

To achieve suitable conditions for the synthesis of spirooxindole 4, various reaction conditions and catalysts have been investigated in the reaction of N-benzylisatin 1a, 4-chloro aniline 2a and 3mercaptopropionic acid $\mathbf{3}$ as a model reaction (table 1 ).

It turned out that the multi-component reactions of 1a, 2a and $\mathbf{3}$ proceeded smoothly in an IL, [bmim] $\left[\mathrm{PF}_{6}\right]$, 1-butyl-3-methylimidazolium hexafluorophosphate, and gave the corresponding spiro[indole3,2' [1,3]-thiazine]-dione (4a). The yield of $\mathbf{4 a}$ increased remarkably with the temperature increasing until $80^{\circ} \mathrm{C}$ (table 1, entries 1-5). Interestingly, of the two ILs studied, namely $[\mathrm{bmim}]\left[\mathrm{PF}_{6}\right]$ and $[\mathrm{bmim}]\left[\mathrm{BF}_{4}\right]$, [bmim] $\left[\mathrm{PF}_{6}\right]$ gave better result (table 1, entries 4 and 6 ), presumably due to its hydrophobic activation activity. It is postulated that water formed 'in situ' from the condensation process is miscible with hydrophilic [bmim $]\left[\mathrm{BF}_{4}\right]$ and thus detained, which prevents completion of the reaction. In contrast, the hydrophobic nature of $[\mathrm{bmim}]\left[\mathrm{PF}_{6}\right]$ would create a microenvironment to drive the equilibrium by extruding water out of the IL phase and thus result in a higher conversion.

The same reaction was also run in several conventional organic solvents and the results are also included in table 1. Comparing with $\mathrm{CH}_{3} \mathrm{CN}$, DMF, EtOH and water, ILs exhibited enhanced reactivity by reducing reaction time and improving the yields significantly. Recovery and reuse of $[\mathrm{bmim}]\left[\mathrm{PF}_{6}\right]$ were also studied, and 1a, 2a and $\mathbf{3}$ were used as model substrates. Upon completion of the condensation process, 4a was obtained by thorough extraction with diethyl ether and the remaining IL phase was recycled in subsequent reactions. Further studies showed

Table 1. Effect of reaction conditions.

\begin{tabular}{lcccc}
\hline Entry & Solvent & Temp $\left({ }^{\circ} \mathrm{C}\right)$ & Time $(\mathrm{h})$ & Yield (\%) \\
\hline 1 & {$\left[\mathrm{bmim}_{[}\left[\mathrm{PF}_{6}\right]\right.$} & $\mathrm{r} \mathrm{t}$ & 10 & 45 \\
2 & {$\left[\mathrm{bmim}_{[}\left[\mathrm{PF}_{6}\right]\right.$} & 50 & 8 & 58 \\
3 & $\left.\left[\mathrm{bmim}_{[}\right] \mathrm{PF}_{6}\right]$ & 70 & 8 & 74 \\
4 & {$\left[\mathrm{bmim}_{[}\left[\mathrm{PF}_{6}\right]\right.$} & 80 & 3 & 90 \\
5 & $\left.\left[\mathrm{bmim}_{[}\right] \mathrm{PF}_{6}\right]$ & 90 & 3 & 90 \\
6 & {$\left[\mathrm{bmim}_{[}\left[\mathrm{BF}_{4}\right]\right.$} & 80 & 3 & 78 \\
7 & $\mathrm{CH}_{3} \mathrm{CN}$ & Reflux & 3 & 62 \\
8 & $\mathrm{DMF}_{9}$ & Reflux & 3 & 60 \\
9 & Ethanol & Reflux & 3 & 65 \\
10 & Water & Reflux & 3 & $\begin{array}{r}\text { Mixture of } \\
\text { products }\end{array}$ \\
& & & & \\
\hline
\end{tabular}


Table 2. Studies on recovery and reuse of $[\mathrm{bmim}]\left[\mathrm{PF}_{6}\right]$.

\begin{tabular}{lcccc}
\hline Round & Time $(\mathrm{h})$ & Temp $\left({ }^{\circ} \mathrm{C}\right)$ & Yield $(\%)$ & IL Recovered \\
\hline 1 & 3 & 80 & 90 & 98 \\
2 & 3 & 80 & 90 & 97 \\
3 & 3 & 80 & 87 & 97 \\
4 & 3 & 80 & 84 & 95 \\
5 & 3 & 80 & 81 & 93 \\
\hline
\end{tabular}

Table 3. Syntheis of spiro[indole-3,2' $[1,3]$-thiazine $]-2,4^{\prime}-$ diones $(\mathbf{4} \mathbf{a}-\mathbf{i})$.

\begin{tabular}{|c|c|c|c|c|c|c|}
\hline Entry & mpound & $\mathrm{R}_{1}$ & $\mathrm{R}_{2}$ & $\begin{array}{l}\text { Time } \\
\text { (h) }\end{array}$ & $\begin{array}{l}\text { Yield } \\
(\%)\end{array}$ & $\begin{array}{l}\text { M.p. } \\
\left({ }^{\circ} \mathrm{C}\right)\end{array}$ \\
\hline 1 & $4 \mathbf{a}$ & $-\mathrm{CH}_{2}-\mathrm{C}_{6} \mathrm{H}_{5}$ & $4-\mathrm{Cl}$ & 3 & 91 & 256 \\
\hline 2 & 4b & $-\mathrm{CH}_{2}-\mathrm{C}_{6} \mathrm{H}_{5}$ & $3-\mathrm{F}$ & 2 & 89 & 274 \\
\hline 3 & $4 c$ & $-\mathrm{CH}_{2}-\mathrm{C}_{6} \mathrm{H}_{5}$ & $3-\mathrm{CF}_{3}$ & 2 & 91 & 250 \\
\hline 4 & $4 d$ & $-\mathrm{CH}_{2}-\mathrm{CH}=\mathrm{CH}_{2}$ & $4-\mathrm{Cl}$ & 3 & 90 & 254 \\
\hline 5 & $4 e$ & $-\mathrm{CH}_{2}-\mathrm{CH}=\mathrm{CH}_{2}$ & $3-\mathrm{F}$ & 3 & 88 & 255 \\
\hline 6 & $4 f$ & $-\mathrm{CH}_{2}-\mathrm{CH}=\mathrm{CH}_{2}$ & $3-\mathrm{CF}_{3}$ & 2 & 86 & 260 \\
\hline 7 & $4 g$ & $-\mathrm{C}_{2} \mathrm{H}_{5}$ & $4-\mathrm{Cl}$ & 2 & 92 & 275 \\
\hline 8 & $4 h$ & $-\mathrm{C}_{2} \mathrm{H}_{5}$ & $3-\mathrm{F}$ & 3 & 88 & 270 \\
\hline 9 & $4 i$ & $-\mathrm{C}_{2} \mathrm{H}_{5}$ & $3-\mathrm{CF}_{3}$ & 2 & 87 & 271 \\
\hline
\end{tabular}

Table 4. Synthesis of spiro[acenaphthylene-1, $2^{\prime}-[1,3]$ thiazine]diones $\mathbf{6 a}-\mathbf{f}$.

\begin{tabular}{lclccc}
\hline Entry & Compound & \multicolumn{1}{c}{$\mathrm{R}$} & $\begin{array}{c}\text { Time } \\
(\mathrm{h})\end{array}$ & $\begin{array}{c}\text { Yield } \\
(\%)\end{array}$ & $\begin{array}{c}\mathrm{M} . \mathrm{p} . \\
\left({ }^{\circ} \mathrm{C}\right)\end{array}$ \\
\hline 1 & $\mathbf{6 a}$ & $2-\mathrm{F}$ & 2 & 84 & 257 \\
2 & $\mathbf{6 b}$ & $3-\mathrm{CF}_{3}$ & 2 & 87 & 227 \\
3 & $\mathbf{6 c}$ & $3-\mathrm{CF}_{3}, 4-\mathrm{Cl}$ & 3 & 88 & 145 \\
4 & $\mathbf{6 d}$ & $4-\mathrm{F}$ & 2 & 87 & 193 \\
5 & $\mathbf{6 e}$ & $4-\mathrm{OCH}_{3}$ & 3 & 85 & 131 \\
6 & $\mathbf{6 f}$ & $4-\mathrm{Cl}$ & 2 & 90 & 205 \\
\hline
\end{tabular}

that the recovered $[\mathrm{bmim}]\left[\mathrm{PF}_{6}\right]$ could be successively recycled for at least five times without obvious loss in its efficiency (table 2). Under the above optimized conditions, we have synthesized several spiro[indole3,2 , [1,3]-thiazine]-2,4'-diones by reaction of various amines and substituted isatins (table 3). Compounds 4a-i are stable solids whose structures were established by IR, ${ }^{1} \mathrm{H},{ }^{13} \mathrm{C}$ NMR and mass spectroscopy and elemental analysis.

Encouraged by the results obtained above, we extended and to further explore the potential of this protocol for synthesis of spiro-heterocyclic compounds, isatin was replaced by acenaphthalene-1,2-dione $\mathbf{5}$ and spiro[acenaphthylene-1,2'-[1,3] thiazine] dione derivatives 6a-f were obtained in good yield under the same reaction conditions (table 4 ).

A plausible mechanism for the formation of the cycloadducts $\mathbf{4}$ is proposed in scheme 3 . Firstly, there is condensation between the isatin and aniline leading to formation of the imine derivative [A] by the loss of water molecule followed by reaction between 3-mercaptopropionic acid and imine derivative yields spiro[indole-3,2'[1,3]-thiazine]-2,4'-dione 4 . Role of the IL may be postulated in terms of some Brønsted acidity due to hydrogen atom of imidazolium cation leading to its interaction with the heteroatoms, thereby increasing polarization and promoting the condensation reaction.

\section{Biological evaluation}

Synthesized compounds were subjected to antimycobacterial efficacy against Mycobacterium tuberculosis H37Rv strain and DNA cleavage activity.

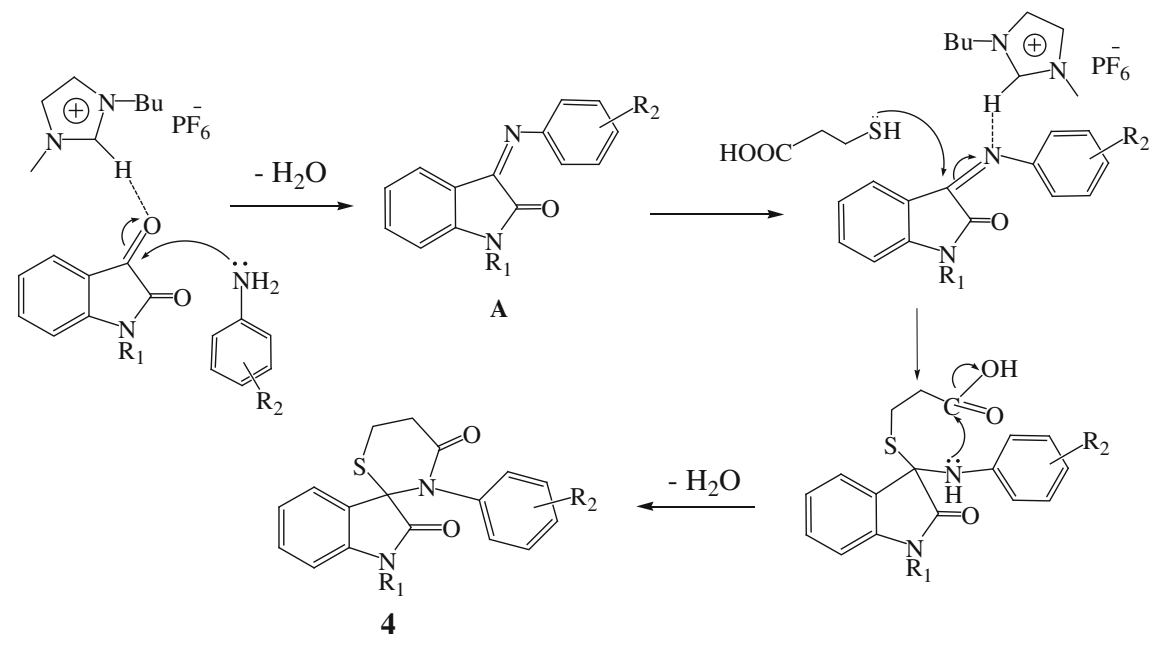

Scheme 3. Plausible mechanism for the synthesis of spiro-thiazine derivatives (4a-i). 


\subsection{In vitro evaluation of antimycobacterial activity}

Minimum inhibitory concentration (MIC) of compounds was determined against M. tuberculosis H37Rv strain by using Lowenstein-Jensen (LJ) medium (conventional method) as described by Rattan. ${ }^{33}$ Determination of MIC of the test compounds against $M$. tuberculosis $\mathrm{H} 37 \mathrm{Rv}$ was performed by LJ agar (MIC) method where primary $(1000,500$ and $250 \mathrm{mg} / \mathrm{ml})$ and secondary $(200,100,62.5,50,25,12.5,6.25$ and $3.25 \mathrm{mg} / \mathrm{ml}$ ) dilutions of each test compound were added to liquid LJ medium and then media were sterilized by inspissation method. A culture of $M$. tuberculosis $\mathrm{H} 37 \mathrm{Rv}$ growing on LJ medium was harvested in $0.85 \%$ saline in Bijou bottles. First stock solution of $2000 \mathrm{mg} / \mathrm{ml}$ concentration of all test compounds was prepared in DMSO. These tubes were then incubated at $37^{\circ} \mathrm{C}$ for $24 \mathrm{~h}$ followed by streaking of $M$. tuberculosis $\mathrm{H} 37 \mathrm{Rv}\left(5 \times 10^{4}\right.$ bacilli per $\left.\mathrm{ml}\right)$. These tubes were then incubated at $37^{\circ} \mathrm{C}$. Growth of bacilli was seen after 12 days, 22 days and finally 28 days of incubation. Tubes having the compounds were compared with control tubes where medium alone was incubated with $M$. tuberculosis H37Rv. Concentration at which no development of colonies occurred or $<20$ colonies was taken as MIC concentration of test compound. The standard strain M. tuberculosis H37Rv was tested with known drug isoniazid. All the compounds exhibited very poor antitubercular activities against M. tuberculosis $\mathrm{H} 37 \mathrm{Rv}$ (table 5).

\subsection{DNA cleavage activity}

A number of studies have shown that clinical efficacies of many drugs correlate with their ability to induce

Table 5. Minimum inhibitory concentrations (MICs, $\mu \mathrm{g} / \mathrm{ml}$ ).

\begin{tabular}{lc}
\hline \multicolumn{2}{c}{ Antimycobacterial activity table } \\
\hline Compound No. & MIC $\mu \mathrm{g} / \mathrm{ml}$ \\
\hline $\mathbf{4 a}$ & 500 \\
$\mathbf{4 c}$ & 63.5 \\
$\mathbf{4 e}$ & 100 \\
$\mathbf{4 f}$ & 500 \\
$\mathbf{4 i}$ & 125 \\
$\mathbf{6 a}$ & 500 \\
$\mathbf{6 b}$ & 150 \\
$\mathbf{6 c}$ & 125 \\
$\mathbf{6 d}$ & 200 \\
$\mathbf{6 e}$ & 63.5 \\
6f & 250 \\
Isoniazid & 0.20 \\
\hline
\end{tabular}

enzyme-mediated DNA cleavage. Inhibitory potency of the test compounds was assessed by comparing the cleavage of DNA by control and the title compound. DNA cleavage experiments were done according to literature. $^{34}$

Nutrient broth (peptone, 10; yeast extract, $5 ; \mathrm{NaCl}$, 10 ; in $(\mathrm{g} / \mathrm{l}))$ was used for culturing the pathogen Staphylococcus aureus. Media $(50 \mathrm{~mL})$ was prepared, and autoclaved for $15 \mathrm{~min}$ at $121^{\circ} \mathrm{C}$ under $15 \mathrm{lb}$ pressures. The autoclaved media was inoculated for $24 \mathrm{~h}$ at $37^{\circ} \mathrm{C}$.

4.2a Isolation of DNA: Fresh bacterial culture $(1.5 \mathrm{~mL})$ is centrifuged to obtain the pellet which was then dissolved in $0.5 \mathrm{~mL}$ of lysis buffer $(100 \mathrm{mM}$ tris $\mathrm{pH}$ 8.0, $50 \mathrm{mM}$ ethylenediaminetetraacetic acid (EDTA), $50 \mathrm{mM}$ lysozyme). To this, $0.5 \mathrm{~mL}$ of saturated phenol was added and incubated at $55^{\circ} \mathrm{C}$ for $10 \mathrm{~min}$, then centrifuged at $10,000 \mathrm{rpm}$ for $10 \mathrm{~min}$ and to the supernatant, equal volume of chloroform: isoamyl alcohol (24:1) and 1/20th volume of $3 \mathrm{M}$ sodium acetate $(\mathrm{pH}$ 4.8) was added. After centrifuging at $10,000 \mathrm{rpm}$ for $10 \mathrm{~min}$, to the supernatant, 3 volumes of chilled absolute alcohol were added. The precipitated DNA was separated by centrifugation and the pellet was dried and dissolved in tris-acetate-EDTA (TAE) buffer $(100 \mathrm{mM}$ tris, $\mathrm{pH} 8.0$ adjusted with glacial acetic acid, $10 \mathrm{mM}$ EDTA) and stored in cold condition.

4.2b Treatment of DNA with the samples: The final compounds $4 \mathbf{a}-\mathbf{i}$ ( $100 \mathrm{mg}$ ) were added separately to the DNA sample. Sample mixtures were incubated at $37^{\circ} \mathrm{C}$ for $2 \mathrm{~h}$.

During agarose gel electrophoresis, ${ }^{34}$ agarose $(200 \mathrm{mg})$ was dissolved in TAE buffer $(25 \mathrm{~mL})(4.84 \mathrm{~g}$ Tris base, $\mathrm{pH}$ 8.0, 0.5 M EDTA/1 L) by boiling. When the gel attained $\approx 55^{\circ} \mathrm{C}$, it was poured into the gel cassette fitted with a comb. The gel was then allowed to solidify. The comb was carefully removed and the gel was placed in the electrophoresis chamber flooded with TAE buffer. DNA sample $(20 \mathrm{~mL}$, mixed with bromophenol blue dye at 1:1 ratio), was loaded carefully into the wells, along with standard DNA marker and constant $50 \mathrm{~V}$ of electricity was passed for around $45 \mathrm{~min}$. The gel was removed and carefully stained with ethidium bromide (ETBR) solution $(10 \mu \mathrm{g} / \mathrm{ml})$ for 10-15 min and the bands were observed under UV transilluminator.

Results are compared with standard DNA marker. All the samples have shown complete cleavage of DNA (figure 1). It was observed from the photograph that compounds $\mathbf{4 a}-\mathbf{i}$ after treatment with DNA (S. aureus) has cleaved it completely. 


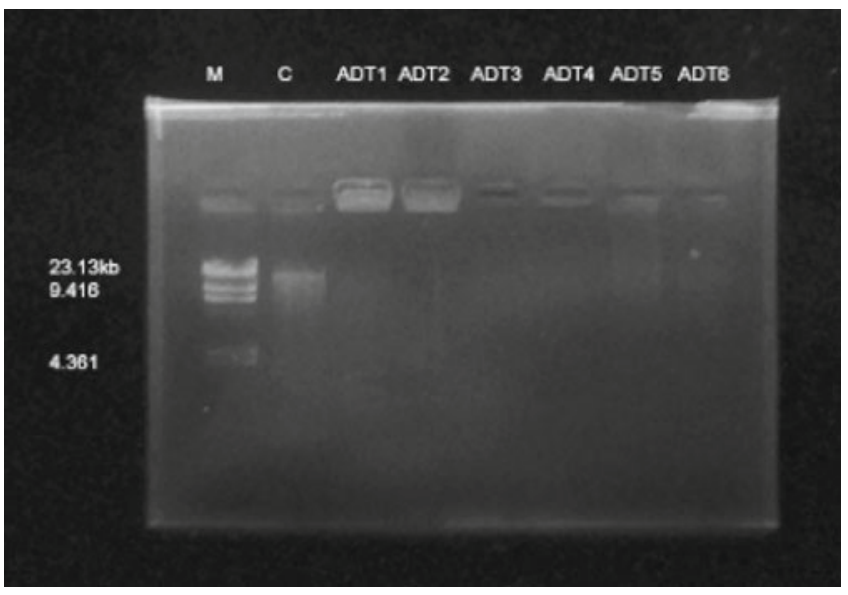

Figure 1. DNA cleavage activity. Lane $\mathrm{M}-$ Standard DNA molecular weight marker ( $\lambda$ DNA Hind III digest), Lane C - Control DNA (untreated sample), Lane ADT1 - 6d, Lane ADT2 - 6b, Lane ADT3 - 6c, Lane ADT4 - 4b, ADT5 - 4d, ADT6 - 4f.

\section{Conclusion}

In conclusion, an efficient and green method has been found for the synthesis of nitrogen and sulphur containing spiro heterocycles via three-component in [bmim] $\left[\mathrm{PF}_{6}\right]$. Features of this procedure include mild reaction conditions, high yields, one-pot, operational simplicity and environmentally benign reactions. DNA cleavage studies revealed that the test compounds in the series have exhibited promising cleavage activity. Based on these results, selected novel compounds are being screened for anticancer activity which will be reported in due course.

\section{Acknowledgements}

Financial assistance from the Council of Scientific and Industrial Research (CSIR), New Delhi is gratefully acknowledged. RS is thankful to CSIR for the award of SRA (No. 13 (8424-A) 2010-Pool). We are also thankful to the Central Drug Research Institute (CDRI), Lucknow for the spectral and elemental analyses.

\section{References}

1. Sun Y, Bi S, Song D, Qiao C, Mu D and Zhang H 2008 Sensors and Actuators B 129799

2. Indumathi T, Fronczek F R and Prasad K J R 2012 J. Mol. Struct. 1016134

3. (a) Gruber B, Kataev E, Aschenbrenner J, Stadlbauer S and Koenig B 2011 J. Am. Chem. Soc. 133 20704; (b) Shao Y, Sheng X, Li Y, Jia Z L, Zhang J J, Liu F and Lu G Y 2008 Bioconjugate Chem. 191840
4. (a) Reifchneider W, Bisabari-Ershadi B, Dripps J E and Bonron J B 1991 U.S. Patent 5,075,293, Chem. Abstr. 116, 1991, 129249f; (b) Rovnyak G C, Narayanan V L and Haugwitz R D U.S. Patent 4,053, 613, 1975; Chem. Abstr. 88, 1978, 22892; (c) Ali S and Alam M 1994 Arch. Pharmacol Res. 17131

5. (a) Zhou H Y, Wu S H, Zhai S M, Liu A F, Sun Y, Li R S, Zhang Y, Ekins S, Swaan P W, Fang B L, Zhang B and Yan B 2008 J. Med. Chem. 51 1242; (b) Ottana R, Carotti S, Maccari R, Landini I, Chiricosta G, Caciagli B, Vigorita M G and Mini E 2005 Bioorg. Med. Chem. Lett. 15 3930; (c) Eriksson B, Kurz G, Hedberg C and Westman J, WO2007010273, 2007

6. Look G C, Schullek J R, Holmes C P, Chinn J P, Gordon E M and Gallop M A 1996 Bioorg. Med. Chem. Lett. 6 707

7. Allen S, Newhouse B, Anderson A S, Fauber B, Allen A, Chantry D, Eberhardt C, Odingo J and Burgess L E 2004 Bioorg. Med. Chem. Lett. 141619

8. (a) Ottana R, Carotti S, Maccari R, Landini I, Chiricosta G, Caciagli B, Vigorita M G and Mini E 2005 Bioorg. Med. Chem. Lett. 15 3930; (b) Gududuru V, Hurh E, Dalton J T and Miller D D 2004 Bioorg. Med. Chem. Lett. 145289

9. Diurno M V, Mazzoni O, Piscopo E, Calignano A, Giordano F and Bolognese A 1992 J. Med. Chem. 35 2910

10. Rawal R K, Tripathi R, Katti S B, Pannecouque $\mathrm{C}$ and DeClercq E 2007 Bioorg. Med. Chem. 153134

11. Campaigne E and Nargund P K 1964 J. Org. Chem. 29 224

12. Barret G C, Kane V V and Lowe G 1964 J. Chem. Soc. 78323

13. Schmidt R R 1972 Synthesis $\mathbf{3 3 3} 7$

14. (a) Kuznetsova L, Ungureanu M I, Pepe A, Zanardi I, Wu X and Ojima I 2004 J. Fluorine Chem. 125 415; (b) Kirsch P 2004 Modern fluoroorganic chemistry, synthesis, reactivity and applications, (New York/Heidelberg: Wiley- $\mathrm{VCH}$ )

15. (a) Quan M L, Lam P Y S, Han Q, Pinto D J P, He M Y, Li R H, Ellis C D, Clark C A, Sun J H, Alexander R S, Bai S, Luettgen J M, Knabb R M, Wong P C and Wexler R R 2005 J. Med. Chem. 48 1729; (b) Abid M and Torok B 2005 Adv. Synth. Catal. 3471797

16. O'Hagan S C, Cobb S L, Hamilton J T G, Cormac D and Murphy C D 2002 Nature 416279

17. Chen I, Safe S and Bjeldanes L 1996 Biochem. Pharmacol. 511069

18. Suzen S and Buyukbingol E 2000 Il Farmaco 55246

19. (a) Buyukbingol E, Suzen S and Klopman G 1994 Il Farmaco 49 443; (b) Suzen S and Buyukbingol E 1998 Il Farmaco 53525

20. (a) Lieberman P M, Wolfler A, Felsner P, Hofer D and Schauenstien K 1997 Int. Arch. Allergy. Immunol. 112 203; (b) Page D, Yang H, Brown W, Walpole C, Fleurent M, Fyfe M, Gaudreault F and Onge S S 2007 Bioorg. Med. Chem. Lett. 226183

21. (a) Cui C -B, Kakeya H, Okada G, Onose R and Osada H 1996 J. Antibiot. 49 527; (b) Cui C -B, Kakeya H and Osada H 1996 Tetrahedron 5212651

22. (a) Khafagy M M, El-Wahas A H F A, Eid F A and ElAgrody A M 2002 Farmaco 57 715; (b) Sebahar P R and Williams R M 2000 J. Am. Chem. Soc. 1225666 
23. Kang T H, Matsumoto K, Murakami Y, Takayama H, Kitajima M, Aimi N and Watanabe H 2002 Eur. J. Pharmacol. 44439

24. Garcia Prado E, Garcia Gimenez M D, De la Puerta Vazquez R, Espartero Sanchez J L and Saenz Rodriguez M T 2007 Phytomedicine 14280

25. Kutschy P, Suchy M, Monde K, Harada N, Maruskova R, Curillova Z, Dzurilla M, Miklosova M, Mezencev R and Majzis J 2002 Tetrahedron Lett. 439489

26. (a) Chen H and Shi D 2011 Tetrahedron 67 5686; (b) Khanna. P, Saxena A, Khanna L, Bhagat S and Jain S C 2009 Arkivoc VII 119; (c) Mashelkar U C, Rane D M and Kenny R S 2008 J. Heterocycl. Chem 45 865; (d) Jain S C, Khanna P, Bhagat S, Jain M and Sakhuja R 2005 Phosphorus, Sulfur Silicon Relat. Elem. 180 1829; (e) Azizian J, Morady A V, Jadidi K, Mehrdad M and Sarrafi Y 2000 Synth. Commun. 30 537; (f) Rajopadhye M and Popp F D 1984 J. Heterocycl. Chem. 21289

27. (a) Houlihan W J, Remers W A and Brown R K 1992 Indoles: Part I. (New York: Wiley); (b) Sundberg R J 1996 The Chemistry of Indoles (New York: Academic Press)

28. (a) Popp F D and Rajopadhye M 1985 J. Heterocycl. Chem. 22 93; (b) Joshi K C, Dandia A and Ahm N 1986 Heterocycle 242479

29. (a) Domling A 2002 Curr. Opin. Chem. Biol. 6 306; (b) Ugi I and Heck S 2001 Comb. Chem. High Throughput Screening 4 1; (c) Zhu J and Bienayme H 2005
Multicomponent reactions (Weinheim: Wiley-VCH); (d) Nicolaou K C, Edmonds D J and Bulger P G 2006 Angew. Chem. Int. Ed. 45 7134; (e) Simon C, Vinod A U, Constantieux T J and Roadriguez K 2004 Eur. J. Org. Chem. 49572004

30. (a) Dzyuba S V and Bartsch R A 2003 Angew. Chem., Int. Ed. 42 148; (b) Wilker J S 2002 Green Chem. 4 73; (c) Welton T 1999 Chem. Rev. 99 2071; (d) Plaquevent J-C, Levillain J, Guillen F, Malhiac C, Gaumont A-C 2008 Chem. Rev. 108 5035; (e) Martins M A P, Frizzo C P, Moreira D N, Zanatta N and Bonacorso H G 2008 Chem. Rev. 108201

31. Joseph T, Sahoo S and Halligudi S B 2005 J. Mol. Catal. A: Chem. 234107

32. (a) Dandia A, Laxkar A K, Singh R 2012 Tetrahedron Lett. 53 3012; (b) Dandia A, Singh R and Bhaskaran S 2011 Green Chem. 13 1852; (c) Dandia A, Jain A K and Bhati D S 2011 Tetrahedron Lett. 52 5333; (d) Dandia A, Parewa V, Jain A K and Rathore K S 2011 Green Chem. 13 2135; (e) Dandia A, Singh R and Bhaskaran S 2010 Ultrason. Sonochem. 17 399; (f) Dandia A, Singh $\mathrm{R}$ and Bhaskaran S 2011 Ultrason. Sonochem. 18 1113; (g) Dandia A, Jain A K, Bhati D S and Sharma G N 2011 Ultrason. Sonochem. 181143

33. Rattan A 2000 Antimicrobials in laboratory medicine. Churchill B I, Livingstone New Delhi, 85

34. Sambrook J, Fritsch E F and Maniatis T 1989 Molecular cloning, A Laboratory Manual, 2nd edn. (Cold Spring Harbor, New York: Cold Spring Harbor Laboratory) 